\title{
Anatomia virtual: atividades extracurriculares desenvolvidas durante a pandemia do COVID-19
}

\section{Virtual anatomy: extracurricular activities developed during the COVID-19 pandemic}

\author{
Célia Cristina Fornaziero ${ }^{1}$ \\ Manuella Fornaziero Marques ${ }^{2}$ \\ Mayara Bocchi ${ }^{3}$ \\ Eduardo Vignoto Fernandes ${ }^{4}$
}

\begin{abstract}
Resumo O objetivo do presente estudo foi propor novas formas de ensino-aprendizagem sobre Anatomia Humana para o curso de Medicina durante o período de pandemia. Este estudo trata-se de um relato de experiência sobre o projeto de ensino "Metodologias aplicadas no Ensino da Anatomia Humana", que explicita metodologias a serem aplicadas em atividades extraclasses como a elaboração de roteiros para aulas práticas, construção de legendas para peças expostas no Museu de Anatomia, uso da impressão 3D na criação de material didático, formas de conservação de peças anatômicas, leitura e escrita científica e ainda o emprego da aromaterapia para possibilitar um ambiente de retorno mais agradável. Para tanto, oito acadêmicos do primeiro ano do curso de Medicina de uma instituição pública, desenvolveram essas atividades orientadas por docentes de Anatomia. O comprometimento dos participantes no projeto pode ser evidenciado na qualidade das atividades desenvolvidas, bem como na assiduidade de cada atividade proposta. Assim, concluímos que o presente estudo pode mostrar inúmeras alternativas de ensino-aprendizagem que podem ser realizadas durante o período pandêmico e continuar motivando os acadêmicos a se manter estudando e contribuindo com sua formação acadêmica.
\end{abstract}

Palavras-chave: Aprendizado “online”. Educação em medicina. Atividades educativas.

Abstract The purpose of this study was to propound contemporary methods of teaching-learning about Human Anatomy for the course of Medicine during the pandemic period. This research is an experience report referring to the teaching project "Methodologies applied in Human Anatomy teaching", which explains procedures to be enforced in extracurricular activities like preparation of scripts for practical classes, construction of subtitles for pieces exhibited at the anatomy museum, using the 3D printer in the creation of teaching materials, techniques to preserve anatomical parts, scientific reading and writing and also the use of aromatherapy to enable a more pleasant return environment. Therefore, eight firstyear academics of the Medicine course of a public institution have developed those activities guided by anatomy professors. The commitment of the project participants can be evidenced in the prospered actions quality as in the attendance of each proposed activity. In consequence, we are allowed to conclude that the present study is able to reveal numerous alternatives of teaching-learning which can

\footnotetext{
${ }^{1}$ Universidade Estadual de Londrina. Departamento de Anatomia, Londrina-PR, Brasil. celiafornaziero@uel.br ${ }^{2}$ Colégio Universitário, Londrina-PR, Brasil. manuellamarques10@gmail.com

${ }^{3}$ Universidade Estadual de Londrina. Departamento de Ciências Patológicas, Londrina-PR, Brasil. mayara_bocchi@hotmail.com

4 Universidade Federal de Jataí. Laboratório de Anatomia Humana e Comparativa, Jataí-Go, Brasil. eduardovignoto@ufj.edu.br
} 


\section{(atnerarius Reflectionis

\section{Volume 17, n.4, ano, 2021}

be performed through the pandemic period and continue motivating academics to remain studying and contributing to their academic formation.

Keywords: Online learning. Medical education. Educational activities.

\section{INTRODUÇÃO}

No final de 2019, um grupo de pessoas com pneumonia de etiologia desconhecida foi observado em Wuhan, China (ZHU et al., 2020). Após um mês, as autoridades sanitárias daquele país alertaram para a existência da doença do novo coronavírus (COVID-19), que causa síndrome respiratória aguda (SARS-COV-2). Em meados de março de 2020, já havia uma pandemia a ser enfrentada por vários países. Em abril de 2020, o número de casos reportados passou de um milhão, distribuídos em 205 países e territórios (WORLDOMETER, 2021).

Por todo o mundo, medidas de restrição foram adotadas na tentativa de atenuar a disseminação do vírus: distanciamento social, uso de máscaras faciais e a utilização de álcool $70 \%$ para higienização das mãos (CHENG et al., 2021). Por conseguinte, a rotina da população foi drasticamente alterada, houve superlotação de hospitais, fechamento de comércio não essencial e de instituições de ensino, cancelamento de viagens e em alguns países, o fechamento das fronteiras, a fim de evitar a aglomeração de pessoas e diminuir a disseminação do vírus.

Diante desse panorama, em maio de 2020, a Organização das Nações Unidas para Educação, Ciência e Cultura estimou que 1,3 bilhão de estudantes, em escala global, de todos os níveis de ensino foram afetados pelas restrições sociais (ORGANIZAÇÃO DAS NAÇÕES UNIDAS PARA A EDUCAÇÃO, A CIÊNCIA E A CULTURA, 2020). As instituições educacionais tiveram que migrar do ensino em sala de aula (presencial), para o ensino remoto. Logo, professores por todo o mundo enfrentaram o desafio de reaprender a trabalhar, por meio da utilização de plataformas virtuais.

Essa condição que influenciou diretamente na ferramenta didática utilizada nas aulas de Anatomia Humana (AH) e no processo de ensino-aprendizagem (ANTONIASSI et al., 2021; SANTOS et al., 2020). Neste contexto, a disciplina de AH, base para grande parte dos cursos de graduação na área de saúde e biológicas (HARMON et al., 2021), precisou ser reinventada para mitigar os possíveis prejuízos na formação acadêmica, assim, professores da área encontraram-se frente a uma nova proposta: como ensinar AH durante a pandemia de COVID19.

Tradicionalmente, a disciplina de AH é cursada ainda no primeiro ano da graduação e os acadêmicos logo percebam que esse conhecimento é imprescindível para o entendimento de 


\section{Citinerarius Refifectionis

\section{Volume 17, n.4, ano, 2021}

outras ciências ao longo do curso e primordial para a sua futura atuação profissional (RUZYCKI et al., 2019). Por ser uma disciplina essencialmente ministrada de forma presencial em laboratórios, foi afetada pelo distanciamento social, impossibilitando as atividades acadêmicas tradicionais, como o uso de prossecção para o aprendizado ou mesmo a impossibilidade da dissecção de peça cadavérica.

Isto posto, pesquisadores do ensino superior elencaram tecnologias para substituir as atividades que são inviáveis durante a pandemia e a comunidade internacional tem reportado a aplicação de diversas experiências com o uso da tecnologia, atribuindo o conceito de Anatomia Virtual (HARMON et al., 2021). Plataformas como Google Meet ${ }^{\circledR}$ foram adotadas por renomadas instituições de ensino superior no Brasil, como na Universidade Estadual de Londrina (UEL).

Essas mudanças na área educacional trouxeram oportunidades de reflexão sobre o processo de ensino-aprendizagem da $\mathrm{AH}$, já que aumentam as chances de interações entre os acadêmicos de várias universidades (HERR e NELSON, 2020), impactando em importantes trocas de experiências de projetos de ensino. Além de proporcionar aos professores a ampliação de seu escopo de materiais didáticos (LONGHURST et al., 2020; WILCHA, 2020).

Com o intuito de contribuir para a melhoria do processo de aprendizagem da AH durante a pandemia causada pelo COVID-19, o presente estudo teve como objetivo destacar alternativas pedagógicas empregadas pelos professores do Departamento de Anatomia da UEL, colaborando com os acadêmicos do curso de Medicina na superação dos desafios e obstáculos dessa nova realidade em sala de aula virtual.

\section{METODOLOGIA}

\subsection{Tipo de estudo}

O presente estudo tratou-se de um relato de experiência sobre o projeto de ensino intitulado: "Metodologias aplicadas no Ensino da Anatomia Humana", cadastrado no sistema UEL sob n ${ }^{0}$ 00837. Para tanto, estratégias de ensino extraclasses foram planejadas, ou seja, além das atividades pré-determinadas pela grade curricular do curso de Medicina, como as aulas teóricas “online” e atividades síncronas para sanar dúvidas antes da avaliação do conteúdo cognitivo; os acadêmicos participantes do projeto tiveram a oportunidade de vivenciar novas estratégias para ampliar o processo de ensino-aprendizagem em $\mathrm{AH}$, bem como suas competências em oralidade e trabalho em grupo. 
Volume 17, n.4, ano, 2021

\subsection{Ensino de AH para o curso de Medicina na UEL (pré e durante pandemia)}

$\mathrm{O}$ atual currículo do curso de Medicina da UEL é baseado em metodologias ativas de ensino-aprendizagem e, em especial, a Aprendizagem Baseada em Problemas (UNIVERSIDADE ESTADAL DE LONDRINA, 2005). Neste contexto, o ensino de AH para esse curso é apresentado durante os três primeiros anos da graduação, por meio de palestras presenciais, cujos temas estão inseridos no contexto do módulo temático que o estudante está cursando, seguidas de aulas práticas em laboratório, utilizando peças cadavéricas por prossecção.

Em conjunto, há discussão tanto dos conteúdos anatômicos como dos assuntos abordados por outras disciplinas formadoras do módulo, em grupos de oito ou nove acadêmicos com um professor tutor, durante o desenvolvimento do módulo temático. Esses grupos tutoriais têm reuniões periódicas duas vezes por semana por um período de oito semanas, média de duração de um módulo temático.

Imprescindivelmente, diante da pandemia de COVID-19, a UEL suspendeu as atividades presenciais em março de 2020 (UNIVERSIDADE ESTADAL DE LONDRINA, 2020). Por conseguinte, foi implantado o cronograma de aulas no formato "online", com as palestras de $\mathrm{AH}$ sendo ministradas em atividades síncronas, por intermédio do Google Meet ${ }^{\circledR}$.

Além dessas atividades, grupos menores de oito estudantes encontravam-se remotamente com seus tutores para discutir temas de ensino abordados no módulo, bem como revisarem a $\mathrm{AH}$. Antes de cada avaliação cognitiva do conteúdo científico do módulo temático, era reservado horário para os acadêmicos tirarem dúvidas com os docentes da $\mathrm{AH}$.

Nesse ambiente virtual (síncrono), os acadêmicos do curso de Medicina poderiam formular perguntas sobre os temas das aulas ou mesmo retomar algum assunto que não foi claramente compreendido durante as palestras. No decorrer do módulo, os conteúdos teóricos de $\mathrm{AH}$ ficavam à disposição dos acadêmicos no Classroom $^{\circledR}$, por meio da gravação das atividades síncronas.

\subsection{Organização e dinâmica do projeto}

Com o decorrer das atividades remotas na UEL, estudantes do curso de Medicina relataram dificuldades de aprendizado em AH em seu formato "online". Ao considerar essa demanda, este projeto de ensino foi iniciado para os acadêmicos do $1^{\circ}$ ano do curso de Medicina da UEL, com intuito de buscar alternativas pertinentes ao desenvolvimento de competências e aprimoramento de conteúdo científico aprendido por esse grupo de estudantes. 


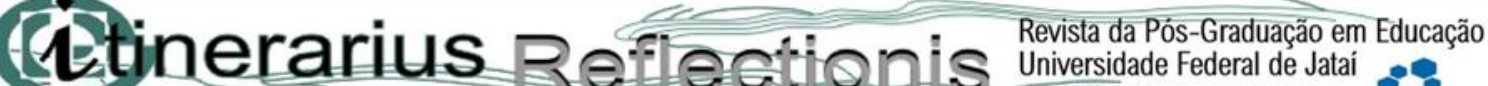

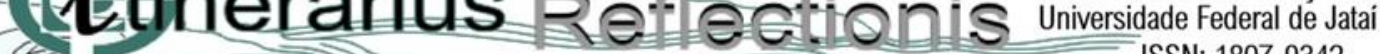 ISSN: 1807-9342}

Volume 17, n.4, ano, 2021

Os oito acadêmicos participantes do projeto contribuíram com as atividades desenvolvidas por cinco professores do Departamento de Anatomia da UEL e um professor da Universidade Federal de Jataí (UFJ). Cada docente ficou responsável por orientar, pelo menos, um estudante dentro de sua área de atuação na Anatomia, assim os participantes do projeto foram distribuídos, segundo suas afinidades, nas subáreas da Anatomia. Dentro dessa organização, cada professor orientador desenvolveu atividades síncronas e assíncronas com seus orientandos (Figura 1).

Figura 1. Projeto de ensino: metodologias aplicadas no ensino da Anatomia Humana.

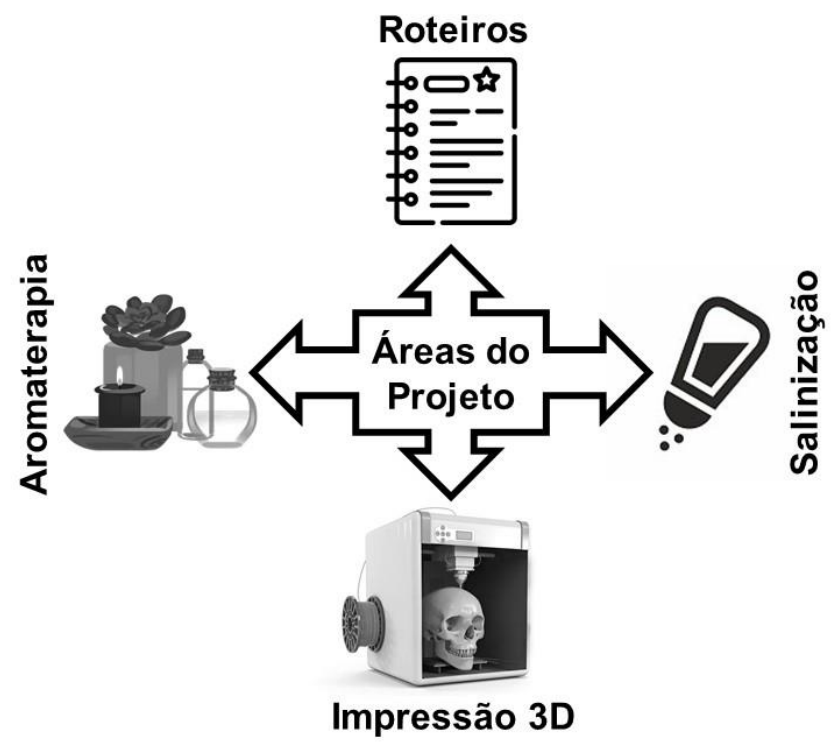

Fonte: (Os autores, 2021).

Legenda: atividades desenvolvidas no projeto: construção de roteiros de aulas práticas; confecção de legendas descritivas e impressão 3D para o museu de Anatomia/UEL; estudo sobre salinização e plastinação de peças anatômicas; estudo do bem-estar do estudante ao retornar em aula prática pós-pandemia por meio de aromaterapia.

Simultaneamente, o grupo se reunia quinzenalmente com a coordenadora do projeto para relatar as atividades desenvolvidas e ter um momento de aprendizado, assistindo a palestras ou participando de debates com professores convidados sobre: construção de material didático, elaboração de artigo científico, plataforma "online" para dinamizar os trabalhos, construção do site da Anatomia, comunicação e preparo de resumos para eventos da área médica. Também, aconteceram discussões sobre metodologias aplicadas no ensino da $\mathrm{AH}$ e análise do perfil do ingressante da UEL. 


\section{RESULTADOS}

Os estudantes responsáveis pela elaboração dos roteiros didáticos para as aulas práticas de $\mathrm{AH}$, desenvolveram materiais didáticos nos seguintes temas: aparelhos locomotor, cardiorrespiratório, urogenital, sistemas digestório e linfático. Cada roteiro foi elaborado e corrigido de acordo com as peças anatômicas disponíveis para visualização no laboratório de AH voltado ao curso de Medicina. Para a construção dos roteiros, os estudantes assistiam às palestras disponibilizadas no Classroom $®$ e faziam seus apontamentos.

A equipe que se dedicou ao museu, reescreveu as legendas das peças anatômicas expostas no Museu de Anatomia da UEL, as quais eram muito antigas e apresentavam alguns erros técnicos. As legendas foram recriadas com o intuito de tornar este museu acessível tanto para visitas virtuais, como para ter acessibilidade em futuras visitas presenciais.

As visitas virtuais acontecerão no site do Departamento de Anatomia da UEL, onde serão disponibilizadas as mesmas imagens das peças expostas com as legendas explicativas gravadas, bem como vídeos e atividades interativas voltadas aos estudantes escolares. No desenvolvimento desta atividade participa um estudante do ensino médio, que contribuiu na elaboração uma escrita mais acessível aos visitantes. Além disso, foi criado um código "Quick Response" (código QR) para todas as legendas e impressões 3D foram disponibilizadas para auxiliar a inclusão de pessoas com deficiência visual nas visitas ao museu.

Outros participantes se dedicaram ao estudo da salinização das peças do laboratório de Anatomia, as quais atualmente são formolizadas. Além do processo de salinização, estudos e levantamento bibliográfico foram desenvolvidos para entender os demais processos de conservação das peças cadavéricas, como a plastinação, com o objetivo de avaliar o custobenefício para à saúde de professores, acadêmicos e técnicos que trabalham diariamente com o formol, bem como, reduzir o gasto com a manutenção do acervo cadavérico da UEL.

Um participante do grupo tem investigado na literatura formas de tornar o retorno às atividades práticas mais confortável aos discentes e docentes, visto que os laboratórios de $\mathrm{AH}$ remetem ao estresse emocional. Na tentativa de proporcionar um ambiente laboratorial mais adequado ao bem-estar de cada indivíduo, estratégias de estudo com aromaterapia, utilizando óleos essenciais de vegetais, tem sido feito a fim de encontrar um óleo mais adequado a este ambiente. 


\section{(atinerarius Refiectionis

Volume 17, n.4, ano, 2021

Com a distribuição dos estudantes em pequenos grupos (até dois acadêmicos), houve um grande avanço dos objetivos pretendidos durante o projeto, pois os professores se dedicaram a um trabalho mais direcionado, conseguindo assim, mesmo com dinâmicas predominantemente "online", criar oportunidades de aprendizado, experiências de desenvolvimento cognitivo e de competências como oralidade e trabalho em equipe.

Além das atividades já supracitadas, vinculadas diretamente as ações do projeto, no primeiro trimestre de 2021, os participantes do projeto também tiveram a oportunidade de aprendizado em escrita científica e apresentação de e-posters em congressos "online" da área médica, sendo que produziram resumos de relatos de experiência no Congresso Catarinense e Paranaense de Educação Médica (CCPEM), cujo tema era educação médica na pandemia de COVID-19. Outros trabalhos foram enviados ao Congresso Mineiro de Educação Médica (COMEM).

Os trabalhos de relato de experiência aprovados para apresentação foram: elaboração de roteiros para o retorno das aulas práticas de $\mathrm{AH}$; desafios do ensino remoto em $\mathrm{AH}$ ocasionado pela pandemia do coronavírus; visitas remotas em tempos de pandemia: acesso virtual ao Museu de Anatomia; implicações do ensino remoto na saúde mental dos discentes de Medicina durante a pandemia de coronavírus; influências do ensino remoto decorrente da pandemia da COVID-19 no aprendizado em uma metodologia ativa e a aromaterapia como aliado do ensino remoto.

Independente do momento da pandemia que restringe atividades presenciais, o projeto tem sido eficiente para o desenvolvimento de trabalho em grupo e tem se mostrado como uma ferramenta de auxílio para o ensino remoto da anatomia, pois o projeto tem atingido seus objetivos de integralizar o estudo da $\mathrm{AH}$ como atividades de desenvolvimento de competências importantes ao acadêmico do curso de Medicina. A assiduidade dos participantes foi de $100 \%$ nas atividades síncronas e a percepção dos professores é que há um comprometimento com o desenvolvimento das tarefas distribuídas a cada semana, somando a qualidade de resposta de cada atividade proposta pelo professor orientador.

\section{DISCUSSÃO}

A pandemia do COVID-19 transformou o modelo de ensino nas instituições educacionais, pois o ensino presencial precisou ser substituído por aulas remotas. Nesse contexto, os professores tiveram que se readequar, investindo tempo para a criação de material didático para as atividades remotas, repensar formas de avaliação na modalidade "online" e, 


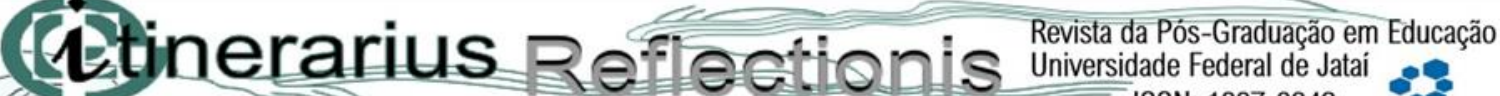

Volume 17, n.4, ano, 2021

simultaneamente, desenvolver suas próprias habilidades tecnológicas (LONGHURST et al., 2020).

Com o intuito de auxiliar no processo de ensino-aprendizagem dos acadêmicos, o presente estudo propôs a elaboração de roteiros de aula, baseados nas palestras "online" de AH, a fim de que estes estudantes conquistem certa independência durante o desenvolvimento das aulas práticas. A eficácia de estratégias complementares e facilitadoras para o ensino de AH já tem sido demonstrada, principalmente quanto se utiliza em paralelo ao roteiro o código QR (DIAS et al., 2020).

A incerteza trazida pela pandemia de COVID-19, bem como as constantes notícias de aumento dos casos positivos, mortes, diminuição do estoque de equipamentos de proteção individual (EPIs) contribuíram para a alta taxa de ansiedade entre os acadêmicos de Medicina (AYITTEY et al., 2020). Esse panorama conduz a uma reflexão de nossas ações no futuro e, a proposta de viabilizar uma visita virtual ao Museu de Anatomia/UEL surgiu para mitigar possíveis transtornos em dias de visitação intensa. Porém, para que isso possa acontecer, todo o planejamento e reestruturação se fez necessário, como a correção e modificação de apresentação das legendas das peças expostas.

Outra questão que não pode ser esquecida é que durante a pandemia, o problema de escassez de cadáver para estudo anatômico ficou mais evidente, trazendo prejuízo ao ensino, pois a falta de peças dissecadas impossibilita o aprendizado das variações anatômicas e não oportuniza o desenvolvimento de compaixão pelo contato com o cadáver (SINGAL, BANSAL e CHAUDHARY, 2020). Também é importante ressaltar os potenciais riscos de transmissão associados ao contato com pessoas que faleceram por conta do COVID-19 (FINEGAN et al., 2020).

Somando-se a escassez cadavérica, os prejuízos para à saúde pelo contato com o formol são amplamente discutidos na literatura, assim para manter uma preservação adequada dos cadáveres já existentes, pensando também, na saúde da equipe em laboratório, este projeto tem trabalhado na substituição do formol por solução salina 30\% (OLIVEIRA, 2014) em todas as peças cadavéricas humanas, condição que já foi implantada na conservação das peças do laboratório de Anatomia Animal.

Segundo Franchi (2020), o desenvolvimento emocional, inteligência, consciência situacional e comportamentos profissionais, bem como sentimentos de empatia são analisados no laboratório de Anatomia por meio de interações com pares, professores, técnicos, acadêmicos e doadores de corpos. Assim, Dubey et al (2020) sugeriu que os alunos do ensino 


\section{(atinerarius Refiectionis

\section{Volume 17, n.4, ano, 2021}

superior são os mais propensos a problemas psicológicos, com altos níveis de ansiedade, em resposta a pandemia de COVID-19. Portanto, este projeto visa minimizar os efeitos do retorno das atividades presenciais, propondo o uso da aromaterapia para mitigar a desconforto dentro de um laboratório de $\mathrm{AH}$, visto que essa área tem conquistado espaço no conceito de qualidade de vida e bem-estar.

Várias alternativas de ensino são apontadas pelos pesquisadores, como o uso de vídeos de dissecção que possam ser encontrados no "Youtube" sob a orientação dos docentes ou podem ser preparados pelos docentes da disciplina de Anatomia, em especial vídeos sobre dissecação (LANGFIELD, COLTHORPE e AINSCOUGH, 2018). Segundo Erolin, Reid e Mcdougall (2019), o ideal para o aprendizado "online" da Anatomia seria a utilização de recursos tecnológicos de realidade virtual.

Por outro lado, ao refletir sobre a realidade social do Brasil, Cunha, Silva e Silva (2020), afirmam que os projetos ou as políticas educacionais precisam ser implantadas baseadas nos indicadores sociais. É necessário considerar que tais estratégias podem ser pouco acessíveis pela maioria das instituições de ensino, assim, os autores apostam na criatividade para propor novas formas de aprender, como o estudo em questão que consegue interação entre os pares com o desenvolvimento de competências inerentes a este processo, juntamente com um aprofundamento dos conceitos da $\mathrm{AH}$.

Não obstante o isolamento social causar inseguranças e dificuldades dentre os discentes, Medeiros et al (2020) propôs uma alternativa de trabalho como a monitoria "online" atuando como uma facilitadora de ensino, como um meio de contribuir com a rotina de estudo e motivação ao aperfeiçoamento, além de facilitar a aquisição de mais conhecimentos acerca da AH. Nesta perspectiva, o presente projeto contribuiu como uma ferramenta de ensinoaprendizagem por meio de atividades com palestrantes da Anatomia, os quais abordaram não somente o conteúdo anatômico como também conhecimento cognitivo para desenvolvimento da oralidade, criação de material didático e construção de conteúdo científico.

O trabalho em grupo é um grande diferencial do projeto, ou seja, o desenvolvimento das atividades propostas conseguiu resgatar a interação entre pares, mesmo com o isolamento social. Segundo (STONE e BARRY, 2019), as interações pessoais, mesmo “online”, não podem ser negligenciadas, já que essa abordagem é essencial para o sucesso do aprendizado remoto, para tanto, faz-se necessário tentar reduzir a distância entre os estudantes por meio da criação de sala de bate-papo ou tutoriais síncronos. 


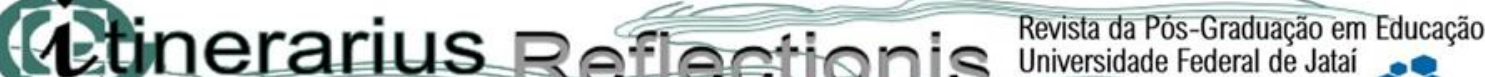

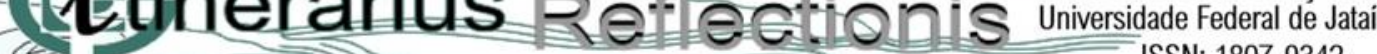

Volume 17, n.4, ano, 2021

Ao considerar que a pandemia impôs mudanças no ensino da Anatomia, Cuschieri e Calleja Agius (2020) defendem que esta pode ser a oportunidade perfeita para revisar currículos, levando em consideração os comentários dos estudantes e os resultados das avaliações nesse período. Em concordância, os autores deste estudo acreditam que a criatividade para dinamizar alternativas de estudo e imprimir oportunidades de interações sociais, mesmo no ensino remoto, sejam essenciais ao processo de aprendizagem.

\section{CONCLUSÕES}

Diante do panorama mundial de pandemia, podemos antever que houve uma grande mudança na forma de ensinar, com uma nova perspectiva de trabalho "online" inserida nas rotinas das aulas. Cabe aos docentes serem criativos em apresentarem propostas de aprendizagem que possam contribuir com o conhecimento do futuro profissional a ser formado.

Nesse sentido, essa nova realidade levantou inúmeras questões educacionais a serem superadas rapidamente pelos docentes e discentes ávidos por aprendizagem, como a disponibilização dos recursos tecnológicos e a conectividade de internet para todos. Dessa forma, o presente estudo pode mostrar inúmeras alternativas de ensino-aprendizagem que podem ser realizadas durante o período pandêmico e continuar motivando os acadêmicos a se manterem estudando e contribuindo com a formação de todos os envolvidos.

\section{REFERÊNCIAS}

ANTONIASSI, Estela et al. Perspectivas de alunos e professores da área da saúde sobre ensino e aprendizado de práticas anatômicas à distância. Brazilian Journal of Animal and Environmental Research, v. 4, n. 1, p. 1483-1499, 2021. DOI:

https://doi.org/10.34188/bjaerv4n1-123

AYITTEY, Foster et al. Economic impacts of Wuhan 2019-nCoV on China and the world. Journal of Medical Virology, v. 92, n. 5, p. 473-475, 2020. DOI:

https://doi.org/10.1002/jmv.25706

CHENG, Xin et al. Gross anatomy education in China during the Covid-19 pandemic: a national survey. Anatomical Sciences Education, v. 14, n. 1, p. 8-18, 2021. DOI: https://doi.org/10.1002/ase.2036

CUNHA, Leonardo; SILVA, Alcineia; SILVA, Aurênio. O ensino remoto no Brasil em tempos de pandemia: diálogos acerca da qualidade e do direito e acesso à educação. Revista Com Censo, v. 7, n. 3, p. 27-37, 2020. Disponível em:

http://www.periodicos.se.df.gov.br/index.php/comcenso/article/view/924. Acesso em: 15 jun. 2021. 


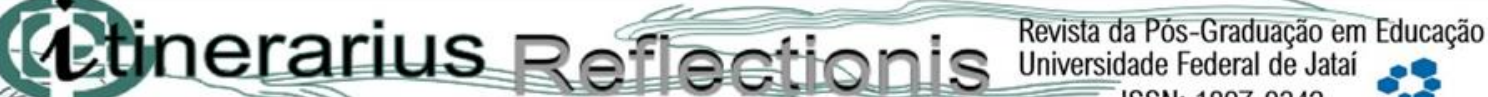 ISSN: 1807-9342}

\section{Volume 17, n.4, ano, 2021}

CUSCHIERI, Sarah; CALLEJA AGIUS, Jean. Spotlight on the shift to remote anatomical teaching during Covid-19 pandemic: Perspectives and Experiences from the University of Malta. Anatomical Sciences Education, v. 13, n. 6, p. 671-679, 2020. DOI:

https://doi.org/10.1002/ase.2020

DIAS, Nícolas et al. A utilização do código de quick response no ensino da anatomia humana do aparelho locomotor. Arquivos de Ciências da Saúde da UNIPAR, v. 24, n. 2, p. 113 116, 2020. DOI: https://doi.org/10.25110/arqsaude.v24i2.2020.7646

DUBEY, Souvik et al. Psychosocial impact of COVID-19. Diabetes \& Metabolic Syndrome: Clinical Research \& Reviews, v. 14, n. 5, p. 779-788, 2020. DOI: https://doi.org/10.1016/j.dsx.2020.05.035

EROLIN, Caroline; REID, Luke; MCDOUGALL, Seaneen. Using virtual reality to complement and enhance anatomy education. Journal of Visual Communication in Medicine, v. 42, n. 3, p. 93-101, 2019. DOI: https://doi.org/10.1080/17453054.2019.1597626

FINEGAN, Oran et al. International Committee of the Red Cross (ICRC): General guidance for the management of the dead related to COVID-19. Forensic Science International: Synergy, v. 2, p. 129-137, 2020. DOI: https://doi.org/10.1016/j.fsisyn.2020.03.007

FRANCHI, Thomas. The impact of the Covid-19 pandemic on current anatomy education and future careers: A student's perspective. Anatomical Sciences Education, v. 13, n. 3, p. 312315, 2020. DOI: https://doi.org/10.1002/ase.1966

HARMON, Derek et al. An Analysis of Anatomy Education Before and During Covid-19: May-August 2020. Anatomical Sciences Education, v. 14, n. 2, p. 132-147, 2021. DOI: https://doi.org/10.1002/ase.2051

HERR, Michael; NELSON, Randall. Administering a stay-at-home dental gross anatomy practical during COVID-19. Journal of Dental Education, p. 1-2, 2020. DOI: https://doi.org/10.1002/jdd.12433

LANGFIELD, Tracey; COLTHORPE, Kay; AINSCOUGH, Louise. Online instructional anatomy videos: Student usage, self-efficacy, and performance in upper limb regional anatomy assessment. Anatomical Sciences Education, v. 11, n. 5, p. 461-470, 2018. DOI: https://doi.org/10.1002/ase.1756

LONGHURST, Georga et al. Strength, weakness, opportunity, threat (SWOT) analysis of the adaptations to anatomical education in the United Kingdom and Republic of Ireland in response to the Covid-19 pandemic. Anatomical Sciences Education, v. 13, n. 3, p. 301-311, 2020. DOI: https://doi.org/10.1002/ase.1967

MEDEIROS, João et al. Desafios do ensino superior durante a pandemia do covid-19: relato de experiência da monitoria de anatomia humana. Brazilian Journal of Development, v. 6 , n. 10, p. 84008-84015, 2020. DOI: https://doi.org/10.34117/bjdv6n10-731 


\section{(1) tinerarius Re

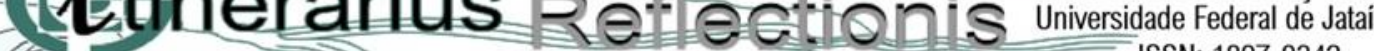 ISSN: 1807-9342}

\section{Volume 17, n.4, ano, 2021}

OLIVEIRA, Fabrício. Assessing the effectiveness of $30 \%$ sodium chloride aqueous solution for the preservation of fixed anatomical specimens: a 5-year follow-up study. Journal of Anatomy, v. 225, n. 1, p. 118-121, 2014. DOI: https://doi.org/10.1111/joa.12185

ORGANIZAÇÃO DAS NAÇÕES UNIDAS PARA A EDUCAÇÃO, A CIÊNCIA E A CULTURA. The United Nations Educational, Scientific and Cultural Organization, Paris, France. 2020. Disponível em: https://en.unesco.org/covid19/educationresponse. Acesso em: 24/05/2021.

RUZYCKI, Stokes et al. Medical education for millennials: How anatomists are doing it right. Clinical Anatomy, v. 32, n. 1, p. 20-25, 2019. DOI: https://doi.org/10.1002/ca.23259

SANTOS, Ana et al. O processo de ensino e aprendizagem de anatomia humana: uma avaliação de estratégias. Ensino, Saude e Ambiente, v. 13, n. 3, p. 362-372, 2020.

SINGAL, Anjali; BANSAL, Agam; CHAUDHARY, Pritiarora. Cadaverless anatomy: Darkness in the times of pandemic Covid-19. Morphologie, v. 104, n. 346, p. 147-150, 2020. DOI: https://doi.org/10.1016/j.morpho.2020.05.003

STONE, Danya; BARRY, Denis. Improving virtual learning interactions: Reducing the transactional distance of online anatomy modules. Anatomical Sciences Education, v. 12, n. 6, p. 686-687, 2019. DOI: https://doi.org/10.1002/ase.1889

UNIVERSIDADE ESTADAL DE LONDRINA. Resolução CEPE n 22/2005. 2005. Disponível em: http://www.uel.br/prograd/?content=pp/pp.html. Acesso em: 24/05/2021.

UNIVERSIDADE ESTADAL DE LONDRINA. Ato Executivo No 22/2020. 2020. Disponível em: https://www.uel.br/com/agenciaueldenoticias/index.php?arq=ARQ_not\&id=30122. Acesso em: $24 / 05 / 2021$.

WILCHA, Robyn-Jenia. Effectiveness of Virtual Medical Teaching During the COVID-19 Crisis: Systematic Review. JMIR Medical Education, v. 6, n. 2, p. e20963, 2020. DOI: https://doi.org/10.2196/20963

\section{WORLDOMETER. Covid-19 Coronavirus Pandemic. Worldometer Information} Technology \& Services, Geneva, Switzerland. 2021. Disponível em: https://www.worldometers.info/coronavirus/. Acesso em: 24/05/2021.

ZHU, Na et al. China Novel Coronavirus Investigating and Research Team. A novel coronavirus from patients with pneumonia in China, 2019. The New England Journal of Medicine, v. 382, n. 8, p. 727-733, 2020. DOI: https://doi.org/10.1056/NEJMoa2001017 